\title{
A PRODUÇÃO DO CONHECIMENTO NO SETOR DOS AGRONEGÓCIOS
}

\author{
L. V. M. BERNARDO * M. J. U. S. FARINHA, E. BINOTTO \\ Universidade Estadual do Oeste do Paraná* e Universidade Federal da Grande Dourados \\ lucianamario@yahoo.com.br*
}

Submetido 22/08/2015 - Aceito 02/11/2018

DOI: $10.15628 /$ holos.2018.6217

\section{RESUMO}

A tecnologia pode ser considerada importante aliada na resolução de problemas em diferentes setores produtivos do Brasil. No caso dos Agronegócios, pode implicar melhoria da produtividade sem o aumento da exploração de áreas produtivas e auxiliar na garantia das questões sanitárias. Para que haja esse processo de criação e inovação é necessário que o conhecimento seja estimulado. O objetivo do artigo é verificar a presença do conhecimento nos espaços destinados aos Agronegócios, verificar especialmente (i) os incentivos governamentais para o desenvolvimento tecnológico, (ii) a produção de maquinários agrícolas e (iii) as indicações geográficas no país. Para tanto foram feitas coletas de dados em diferentes sites governamentais e no anuário da Associação Nacional dos Fabricantes de Veículos
Automotores. Os resultados indicam que existem programas e incentivos financeiros para o desenvolvimento tecnológico, sendo que a maioria das atividades está condizente com as tendências tecnológicas globais. Enfatiza-se que as atividades da produção e da comercialização dos maquinários agrícolas se destacam, em 2014, na Região Sul do país. Com relação às Indicações Geográficas, o ano de 2012 concentra o maior número de Indicações aceitas, sobressaindo-se a produção de vinhos. Implícita ou explicitamente, o conhecimento está presente e influencia a continuidade e os avanços nos elementos tecnológicos que podem positivamente alterar o contexto.

PALAVRAS-CHAVE: Produção de conhecimento, Incentivos governamentais, Desenvolvimento tecnológico, Indicações geográficas de produção.

\section{ABSTRACT}

The technology can be considered important ally in solving problems in different productive sectors of Brazil. For Agribusiness, may involve improving productivity without increasing the exploitation of productive areas and assist in ensuring the health issues. So there is this process of creation and innovation is needed that knowledge is stimulated. The paper aims to verify the presence of knowledge in spaces intended for Agribusiness, check especially (i) government incentives for technology development, (ii) the production of agricultural machinery and (iii) the geographical indications in the country. For both data collections were made at various government sites and in the yearbook of the National Association of
Automobile Manufacturers. The results indicate that there are programs and financial incentives for technology development, and most activities are consistent with the global technological trends. It is emphasized that the activities of the production and marketing of agricultural machinery stand out in 2014 in the southern region of the country. With respect to geographical indications, the year 2012 has the largest number of accepted indications, sticking to wine production. Implicitly or explicitly, knowledge is present and influences the continuity and advances in technological elements that can positively change the context.

KEYWORDS: Production knowledge, Government incentives, Technological development, Geographic production indications. 


\section{INTRODUÇÃO}

Os avanços tecnológicos influenciam diferentes mudanças na sociedade, de modo a que essas informações e conhecimentos venham a ser nela reconhecidos como característicos (TAVARAYAMA; MARTINS, 2012). Denota-se que o progresso neste novo século está condicionado à transformação do conhecimento em tecnologias, mas tecnologias que possam trazer vantagens coletivas (ROCO; BAINBRIDGE, 2013).

No que se refere à produção tecnológica do Brasil, a Constituição Federal vigente aborda, no artigo 218, a exigência de que esteja direcionada às questões problemáticas do país, dando ênfase ao sistema produtivo que privilegie as questões nacionais e regionais (BRASIL, 1988).

Para esses aspectos, Scudeler e Oliveira (2013) relacionam também as questões sociais e econômicas, observando a importância das patentes nesse processo de desenvolvimento tecnológico. Para isso foi criado o Instituto Nacional da Propriedade Intelectual, para as indicações estatísticas dos elementos de interesse dos inventores do país.

No caso dos agronegócios, a utilização da tecnologia tornou-se fundamental para a administração dos negócios, pois pode auxiliar no controle dos custos da produção de maneira a reduzi-los a partir da gestão de tecnologia. Esse é justamente o papel da gestão de tecnologia, o de envolver o modo como os recursos destinados à produção serão aplicados e o conhecimento do valor desses recursos, compreendendo, assim, aqueles que são próprios ou de terceiros (COMPANHIA NACIONAL DE ABASTECIMENTO, 2010).

Para Nascimento et al. (2014), as práticas relacionadas à gestão de tecnologia utilizada na propriedade podem positivamente influenciar os resultados do uso desses elementos, isso devido ao aperfeiçoamento das técnicas de produção, bem como ao uso de métodos propícios ao contexto. Essas práticas significam também a inserção de novos conhecimentos na unidade, o que proporciona vantagens competitivas, benefícios na qualidade dos produtos e aumento na produção.

No caso da produção agrícola, as mudanças ocorridas a partir do final do século XX disponibilizaram à agricultura novas ferramentas tecnológicas, sejam as destinadas ao solo, sejam as destinadas ao plantio, à colheita e à comercialização, que, aliadas aos softwares de gerenciamento, integraram o produtor ao cenário empresarial, pois esse produtor precisa ter mecanismos para administrar as instabilidades que inferem no setor (REDIVO et al., 2008).

O investimento financeiro feito pelo governo, com relação às pesquisas destinadas à produção agrícola, torna-se cada vez mais necessário. Inovações biológicas e procedimentos igualitários para esta nova produção devem fazer parte do planejamento do país. As mudanças climáticas poderão afetar a agricultura e, consequentemente, a economia, caso o país não esteja preparado tecnologicamente para as novas características climáticas criadas (ROCHA, 2014).

A engenharia responsável pelo desenvolvimento de maquinário agrícola acredita que houve, no decorrer da história humana, avanços tecnológicos que possivelmente prosperarão no decorrer das próximas décadas, isso devido às potencialidades que o setor oferece, bem como devido à sua importância no cenário mundial para a produção alimentar e considerando que 
poderá oferecer novos implementos agrários, capazes de obter desempenhos melhores do que aqueles já utilizados (JONGENSEN, 2012).

Deste modo, o propósito do estudo é verificar a relação do conhecimento no agronegócio decorrente de incentivos governamentais para o desenvolvimento tecnológico, a produção de maquinários agrícolas e as Indicações Geográficas no Brasil.

\section{CONHECIMENTO E TECNOLOGIA: UMA RELAÇÃO IMPACTANTE NA SOCIEDADE}

Por meio das tecnologias integradas à informação, a criação de ambientes diferenciados de aprendizagem foi formalizada na sociedade. Não mais se restringem esses ambientes às unidades escolares de ensino-aprendizagem. Agora esses ambientes diferenciados podem ser materializados em espaços distintos, conforme a intenção do indivíduo ou virtuais, por meio do uso da internet (GADOTTI, 2005).

Atualmente, por meio do acesso às informações, o conhecimento pode ser disseminado entre os indivíduos com maior facilidade. As tecnologias vinculadas à internet proporcionaram que esses dados fossem irradiados com muito maior alcance popular e em um menor tempo. Mesmo assim, porém, a integração entre o indivíduo e o conhecimento proveniente desses ambientes digitais exige que o primeiro saiba como utilizar essas ferramentas tecnológicas (DIAS, 2012; BORGES et al., 2013).

A tecnologia impacta na sociedade de modo a indicar mudanças em diferentes aspectos, contemplando, por exemplo, o comportamento social dos indivíduos, assim como influencia a produção de bens e serviços (VAZ et al., 2009). Nesse contexto, a criatividade das pessoas passa a ser aguçada e utilizada de modo a proporcionar ganhos financeiros. Considerando, também, a existência de profissionais com facilidade em tomar decisões, qualificados, experientes e com informações acessíveis, então esses componentes possibilitam que problemas complexos de diferentes áreas sejam resolvidos (FLORIDA, 2011).

Nesse sentido, a sociedade deve observar algumas situações que precisam ser garantidas aos indivíduos, como: o acesso democrático a diferentes fontes de informação e pessoas com habilidades que proporcionem que a informação se torne conhecimento, isso a partir de valores intrínsecos à coletividade, à criatividade, à cooperação, entre outros, tendo o objetivo de inovar considerando os diferentes resultados e as diferentes consequências das ações tomadas (COUTINHO; LISBÔA, 2011). Isso está de acordo com os objetivos presentes na sociedade do conhecimento, que são aprender a conhecer, aprender a fazer, aprender a viver em comum, aprender a ser. Os mesmos objetivos refletem o comportamento das pessoas frente ao conhecimento, iniciando pela compreensão, continuando com aplicação em diferentes situações, cooperação e a constituição das características individuais (ESCOLA, 2005).

A influência da tecnologia na sociedade tangencia seu desenvolvimento de modo a definir o seu progresso ou não. Denota-se que as modificações sociais estão vinculadas à tecnologia, de modo a integrar esses elementos, resultando que um se altere conforme o outro (CARVALHO, 1997; BAZZO, 2012). 


\section{A PRODUÇÃO DO CONHECIMENTO TECNOLÓGICO E SEU REGISTRO}

As primeiras indicações de tecnologia surgiram quando o homem passou a querer registrar suas ações, isso com o intuito de torná-las aparentes à comunidade, mas sem delas perder a propriedade. Assim, as linguagens verbais e não verbais criadas de modo a suprir essa necessidade humana de comunicação podem ser percebidas como uma tecnologia criada e que se destaca perante o contexto histórico (FELIPE, 2012).

O surgimento das tecnologias proporcionou que o conhecimento humano fosse exposto, isso logo implicando um aumento de experiências e pesquisas, de modo a contribuir nas integrações dos saberes (WELTER; CASTRO, 2013). Além das experiências, essas tecnologias ampliaram também as relações humanas, isso de modo a formalizar, enfim, redes de comunicação a partir da constituição de comunidades virtuais (CASTELLS, 2000).

O interesse do governo, nessa questão da produção do conhecimento, proveniente de organizações destinadas a esse fim, é conseguir interagir com os meios de produção da sociedade, assim objetivando que haja avanços no desenvolvimento econômico (NEGRI; CAVALCANTE, 2013).

Desse modo, inserido no contexto estão as patentes, os registros necessários à identificação e à valorização daqueles que desenvolveram determinados inventos. É necessário observar, no que se refere às informações sobre essas tecnologias, que o acesso a elas está mais facilitado, o que significativamente contribui para possíveis análises necessárias para a continuidade de pesquisas posteriores e ao uso dessas inovações (BRASIL, 1996; SILVA et al., 2013).

As tecnologias destinadas à produção de alimentos são desenvolvidas para atender a demandas por determinados tipos de consumo característicos dos costumes da população, bem como buscam adaptar as mudanças nos hábitos alimentares das pessoas. Devido a essas características e ao adicionar o crescimento populacional mundial que influenciará o consumo, esse mercado está propenso a expandir-se tecnologicamente (CAJAVILCA et al., 2014).

No caso das inovações, criadas a partir das necessidades encontradas no campo, compreende-se como foram fundamentais para o desenvolvimento agrícola do Brasil. Se esse processo atingiu os resultados esperados com relação à ampliação da produção, isso foi devido à aprendizagem contida no contexto, aprendizagem que influenciou o uso adequado das tecnologias. Sem elas e o uso adequado delas, as consequências poderiam não ser tão eficazes (OLIVEIRA et al., 2012).

A capacidade inovativa de um empreendimento está vinculada ao conhecimento que seus colaboradores possuem, ao perfil organizacional voltado ao planejamento, implantação e continuidade de estruturas e sistemas que possibilitem a gestão do conhecimento (NOWACKI; BACHNIK, 2015). Essa gestão é a maneira utilizada pelos empreendimentos para criar riqueza por meio do conhecimento presente nas organizações (BUKOWITZ; WILLIAMS, 1999).

Além disso, denota-se que o acesso a informação é um insumo estratégico para o processo de decisão e que pode refletir como uma vantagem competitiva no espaço de negociações do meio rural (FALÉCO; JORGE, 2017). Principalmente se for considerado a alta competitividade 
existente nos mercados de produtos agrícolas, tendo em vista que estes ultrapassam as fronteiras nacionais e as comercializações podem ser realizadas internacionalmente. Esta característica, influenciou a dinâmica das organizações (ARAÚJO, 2013; SILVA et al., 2016).

\section{A PRESENÇA DAS TECNOLOGIAS AGRÁRIAS NA PRODUÇÃO DO CAMPO}

O aumento do uso de tecnologias nos setores dos Agronegócios, a partir da Revolução Tecnológica da década de 1970, ainda influencia o desenvolvimento de técnicas, ferramentas e elementos tecnológicos. Essas características, porém, quando comparadas a cenários que representam alguns países desenvolvidos e outros emergentes, indicam a necessidade de avanços do Brasil, de modo que possibilite o nivelamento com esses outros países (CRUVINEL et al., 2008).

As melhorias tecnológicas agrárias no Brasil foram impulsionadas por três elementos: (i) a instalação de multinacionais direcionadas às atividades do setor, (ii) programas governamentais que visaram o incentivo à aquisição de tecnologias do campo e (iii) criação da Empresa Brasileira de Pesquisa Agropecuária - EMBRAPA, com o intuito do desenvolvimento de pesquisas para o setor (SANTOS, 2014).

Essa possibilidade de auxílio tecnológico na produção, através de máquinas e implementos, trouxe ao setor agrário novas características com relação às técnicas adotadas na produção, à oferta de produtos ao mercado globalizado e à mão de obra a ele vinculada. Essa tendência tornase maior e implica o uso de tecnologias cada vez mais avançadas (VIAN; ANDRADE JÚNIOR, 2010).

No caso da agropecuária do país, Oliveira et al. (2012) indicam um setor competitivo globalmente, tendo como um dos elementos responsáveis a inovações criadas e aplicadas para o progresso das cadeias produtivas relacionadas, o que implica alterações no contexto econômico que passa a ser embasado no conhecimento.

Por outro lado, existem alguns entraves no uso de tecnologias no setor agrário brasileiro, como dificuldades sociais e econômicas de acesso e uso das ferramentas tecnológicas desenvolvidas para a melhoria do contexto das pequenas propriedades. Considera-se, ainda, o excesso de elementos produzidos para sanar um grupo de mesmas dificuldades, enquanto lacunas não são abordadas na confecção de materiais tecnológicos, entre outros (BAMBINI et al., 2013).

Ao se observar o contexto global, para a Food and Agriculture Organization of the United Nations - FAO (2012), o desenvolvimento da tecnologia com relação ao cultivo das produções, a maneira como está sendo tratado o solo, as inovações referentes aos adubos químicos e a modificação genética dos grãos, tudo isso influenciou positivamente para o aumento produtivo, o que auxilia na redução da fome mundial, pois foram obtidos resultados produtivos maiores sem o aumento das áreas cultiváveis.

O uso da mecanização agrária contribui para com a qualidade da produção e sua eficiência. A aquisição desses elementos tecnológicos pode estar vinculada a diferentes componentes, como energia, qualidade do maquinário, impacto ambiental, entre outros. Destaca-se que, a partir da mecanização, um país pode modernizar sua produção agrícola auxiliando no desenvolvimento das atividades agrárias (ZHOU et al., 2011). 


\section{METODOLOGIA}

Esta investigação pode ser considerada descritiva, documental e quantitativa. Seu desenvolvimento consta com coleta de dados nos sites do Banco Nacional de Desenvolvimento Econômico e Social - BNDES com relação aos investimentos e financiamentos destinados a tecnologias agrárias.

Também foram utilizadas as informações coletadas no site do Ministério da Agricultura Pecuária e Abastecimento - MAPA, através de indicações das tendências dos agronegócios no aspecto da evolução tecnológica relacionado aos programas desenvolvidos pelo Ministério de Ciências e Tecnologia - MTC.

O Anuário da Indústria Automobilística Brasileira, divulgado no ano de 2015, também contém informações referentes ao ano de 2014, informações da responsabilidade da Associação Nacional dos Fabricantes de Veículos Automotores - ANFAVEA e contemplando dados que representam a produção e comercialização da produção de maquinários agrícolas.

Também, ainda, o Instituto Nacional de Propriedade Intelectual - INPI, no que se refere às Indicações Geográficas e às Patentes, apresenta informações quanto ao ambiente dos agronegócios. Todos os dados foram coletados em janeiro do respectivo ano e estão organizados em quadros que constituem os resultados da pesquisa, com informações de diferentes entidades e fazendo uso de percentuais para indicar diferenças de valores em anos em que a coleta de informação foi feita.

\section{RESULTADOS E DISCUSSÕES}

\subsection{O BNDES e o Apoio Financeiro à Modernização da Produção dos Agronegócios}

Devido à influência dos agronegócios na economia brasileira, o BNDES incentiva o desenvolvimento das chamadas cadeias produtivas, fazendo-o mediante o crédito BNDES Finem e mais alguns programas existentes devido à sua área de atuação na agropecuária, tratando-se de programas com diferentes linhas de financiamento. Isso significa dar continuidade ao papel do governo como descrito por Santos (2014), para que haja o desenvolvimento tecnológico no país.

O Quadro 01 traz informações sobre as linhas de financiamento desse produto e seus respectivos propósitos:

Quadro 1: Linhas de financiamento aos agronegócios do programa BNDES Finem

\begin{tabular}{|c|l|}
\hline LINHA DE CRÉDITO & \multicolumn{1}{c|}{ OBJETIVO } \\
\hline $\begin{array}{c}\text { Linha de Apoio à } \\
\text { Agropecuária }\end{array}$ & $\begin{array}{l}\text { Dar apoio a propostas que objetivem à implantação, modernização e expansão de } \\
\text { empresas do setor. }\end{array}$ \\
\hline $\begin{array}{c}\text { Projetos de Eficiência } \\
\text { Energética }\end{array}$ & $\begin{array}{l}\text { Contribuir para a economia de energia, aumento da eficiência do sistema energético ou } \\
\text { substituição de combustíveis de origem fóssil. }\end{array}$ \\
\hline $\begin{array}{c}\text { Aquisição de Bens de } \\
\text { Capital }\end{array}$ & Aquisição de bens de capital associada a planos de investimentos. \\
\hline $\begin{array}{c}\text { Aquisição de Bens e } \\
\text { Serviços Importados }\end{array}$ & $\begin{array}{l}\text { Importação de máquinas e equipamentos sem similar nacional, associada a planos de } \\
\text { investimentos. }\end{array}$ \\
\hline BNDES Automático & Implantação, expansão e modernização de empreendimentos. \\
\hline BNDES Finame Agrícola & Aquisição de máquinas e equipamentos agrícolas novos, de fabricação nacional. \\
\hline BNDES Finame Leasing & Aquisição de máquinas e equipamentos novos em operações de arrendamento \\
\hline
\end{tabular}




\begin{tabular}{|c|l|}
\hline \multicolumn{1}{|c|}{ Cartão BNDES } & $\begin{array}{l}\text { Crédito rotativo, pré-aprovado, para aquisição de produtos, insumos e serviços } \\
\text { credenciados no Portal de Operações do Cartão BNDES, direcionado às micro, pequenas } \\
\text { e médias empresas. }\end{array}$ \\
\hline BNDES Limite de Crédito & $\begin{array}{l}\text { Crédito rotativo para o apoio a empresas ou grupos econômicos já clientes do BNDES e } \\
\text { com baixo risco de crédito. }\end{array}$ \\
\hline $\begin{array}{c}\text { BNDES Empréstimo- } \\
\text { Ponte }\end{array}$ & $\begin{array}{l}\text { Financiamento a um projeto, concedido em casos específicos, para agilizar a realização } \\
\text { de investimentos por meio da concessão de recursos no período de estruturação da } \\
\text { operação de longo prazo. }\end{array}$ \\
\hline BNDES Project finance & $\begin{array}{l}\text { Engenharia financeira suportada contratualmente pelo fluxo de caixa de um projeto, } \\
\text { servindo como garantia os ativos e recebíveis desse mesmo empreendimento. }\end{array}$ \\
\hline BNDES Fianças e Avais & $\begin{array}{l}\text { Prestação de fiança e avais pelo BNDES com objetivo de diminuir o nível de participação } \\
\text { nos projetos financiados }\end{array}$ \\
\hline
\end{tabular}

Fonte: BNDES (2015).

Identifica-se que, destas 12 linhas de financiamento, linhas às quais as empresas relacionadas aos agronegócios podem ter acesso, 7 linhas estão relacionadas à aquisição de tecnologias ou à modernização do empreendimento. Ou seja, $58 \%$ das possibilidades de investimentos no setor provenientes desse produto do BNDES destinam-se à tecnologia.

No caso dos programas, o Quadro 02 identifica quais estão em vigência e seus objetivos, isso tendo em vista que $60 \%$ estão destinados à implantação ou à renovação de tecnologias.

Quadro 2: Programas de financiamento aos agronegócios do BNDES.

\begin{tabular}{|c|l|}
\hline LINHA DE CRÉDITO & \multicolumn{1}{c|}{ OBJETIVO } \\
\hline BNDES Proaquicultura & $\begin{array}{l}\text { Investir na construção, expansão e modernização de capacidade produtiva dos } \\
\text { produtores e da indústria processadora; ao capital de giro; e a iniciativas voltadas } \\
\text { para modernização ou implementação de melhorias na estrutura organizacional, } \\
\text { administrativa, de gestão, comercialização, distribuição e de logística. }\end{array}$ \\
\hline BNDES Cerealistas & $\begin{array}{l}\text { Desenvolver e modernizar o setor de armazenagem nacional efetuado por empresas } \\
\text { comerciais cerealistas nacionais. }\end{array}$ \\
\hline BNDES Prorenova & $\begin{array}{l}\text { Aumento da produção de cana-de-açúcar no país, por meio do financiamento à } \\
\text { renovação e implantação de novos canaviais. }\end{array}$ \\
\hline BNDES PASS & $\begin{array}{l}\text { Financiamento à estocagem de álcool etílico combustível pelas empresas do setor } \\
\text { sucroalcooleiro. }\end{array}$ \\
\hline $\begin{array}{c}\text { Programas do Governo } \\
\text { Federal administrados } \\
\text { pelo BNDES }\end{array}$ & $\begin{array}{l}\text { Opções de financiamento destinadas a produtores rurais e a suas associaçães e } \\
\text { cooperativas, com o objetivo de financiar investimentos em modernização, } \\
\text { reflorestamento, capitalização de cooperativas, dentre outras finalidades mais } \\
\text { específicas. }\end{array}$ \\
\hline
\end{tabular}

Fonte: BNDES (2015).

Não exclusivos aos agronegócios, mas que também poderão estar vinculados ao setor, existem os programas de inovação e do meio ambiente. Esses programas podem via a contribuir para que a tecnologia e, assim, o conhecimento, estejam presentes nas produções do setor, de modo a existir uma produção sustentável que contribua com as necessidades mundiais, conforme o elencado pela FAO (2012).

No que se refere à liberação de recursos do Sistema BNDES para a comercialização de maquinário e equipamentos agrícolas nacionais, o Quadro 03 indica os valores do período de 2003 a 2013, intervalo esse escolhido devido à disponibilidade de informações. 
Quadro 3: Liberação de recursos do BNDES para a aquisição de maquinários e equipamentos agrícolas

\begin{tabular}{|c|c|c|}
\hline ANO & VALORES (R\$ MILHÕES) & VARIAÇÃO PERCENTUAL (\%) \\
\hline 2003 & $2.894,25$ & --- \\
\hline 2004 & $4.585,69$ & 158,44 \\
\hline 2005 & $2.306,41$ & $(49,70)$ \\
\hline 2006 & $1.719,62$ & $(25,45)$ \\
\hline 2007 & $2.643,43$ & 153,72 \\
\hline 2008 & $3.338,53$ & 126,30 \\
\hline 2009 & $3.316,20$ & $(0,67)$ \\
\hline 2010 & $7.189,40$ & 215,34 \\
\hline 2011 & $6.944,50$ & $(3,00)$ \\
\hline 2012 & $8.018,50$ & 115,46 \\
\hline 2013 & $14.384,30$ & 179,38 \\
\hline TOTAL & 50.151 & \\
\hline
\end{tabular}

Fonte: BNDES (2015).

Ao longo do período se destaca que o fluxo da liberação dos recursos não obedece a uma constante, pois os valores variam e não seguem um aumento ou redução gradual. Denota-se a importância destacada por Zhou et al. (2011) sobre a mecanização da produção, o modo como pode influenciar a produção agrícola de um país.

\subsection{As tendências tecnológicas dos agronegócios no Brasil}

A evolução tecnológica é um processo necessário à continuidade do desenvolvimento dos países. Carvalho (1997) e Bazzo (2012) observaram que esse processo pode influenciar o progresso dessas unidades e delinear o modo como decorrerão as atividades para isso. Mesmo assim, porém, a indicação feita por Coutinho e Lisbôa (2011) contribui nos aspectos sociais desse contexto, de modo que essas tecnologias estejam democraticamente acessíveis a toda população.

Esse processo de inovação, do ponto de vista de Vaz et al. (2009), pode influenciar o comportamento humano trazendo aos indivíduos características antes não presentes no cotidiano. Por outro lado, as considerações de Florida (2011) indicam a positividade desse processo, devido, entre outros fatores, à existência de liberdade criativa que auxilia na resolução de problemas complexos.

O Quadro 04, remete-se a importância da tecnologia no contexto dos agronegócios proporcionou que o MAPA organizasse elementos pertencentes a essa evolução:

\begin{tabular}{|c|c|}
\hline $\begin{array}{l}\text { TENDÊNCIAS } \\
\text { GLOBAIS }\end{array}$ & TENDÊNCIAS DOS AGRONEGÓCIOS \\
\hline \multirow{11}{*}{ Evolução Tecnológica } & Aumento da produtividade agropecuária \\
\hline & Aumento da mecanização agrícola \\
\hline & Aumento do uso de biocombustíveis \\
\hline & Diminuição na oferta de mão de obra no campo \\
\hline & Adoção de sistemas produtivos sustentáveis \\
\hline & Desenvolvimento da biotecnologia/biossegurança \\
\hline & Zoneamento de produção \\
\hline & Crescimento na produção de orgânicos \\
\hline & Agricultura de Precisão \\
\hline & Propriedade Intelectual na Agricultura \\
\hline & Alimentos funcionais \\
\hline
\end{tabular}




\begin{tabular}{|l|l|}
\hline & Especialização de produtos para atender a nichos de mercado \\
\cline { 2 - 3 } & Melhor organização da cadeia produtiva \\
\cline { 2 - 2 } & Aumento das exigências em relação à inocuidade, à segurança e à qualidade dos alimentos \\
\cline { 2 - 2 } & Redução dos preços agrícolas (commodities). \\
\hline
\end{tabular}
Fonte: MAPA (2015).

As tendências elencadas abrangem diferentes áreas de atuação dos agronegócios. Há em comum, nos itens elencados, a necessidade de conhecimento para o alcance dessas propensões, pois Welter e Castro (2013) compreendem que a tecnologia é um indicativo das experiências e das pesquisas e tem a finalidade de resolver problemas através das compreensões provenientes das práticas e dos compartilhamentos entre produtores.

Além disso, referente ao Brasil, o MCT possui programas direcionados à inovação e às tecnologias para os agronegócios. No Quadro 05 estão elencados os programas, um por um, com seu respectivo objetivo:

\begin{tabular}{|c|c|}
\hline PROGRAMAS & OBJETIVOS \\
\hline $\begin{array}{l}\text { Pesquisa, Desenvolvimento e Inovação } \\
\text { em Alimentos }\end{array}$ & $\begin{array}{l}\text { Disponibilizar conhecimentos, tecnologias, materiais e sistemas } \\
\text { tecnológicos para incrementar conhecimentos estruturantes e } \\
\text { promover a inovação no agronegócio com foco em segurança de } \\
\text { alimentos e novas frentes abertas pela tecnologia de alimentos. } \\
\text { Alimentos de qualidade, nutracêutica e alimentos funcionais; processos } \\
\text { de rastreabilidade; inocuidade relativa a resíduos químicos e biológicos; } \\
\text { e biossegurança alimentar. }\end{array}$ \\
\hline $\begin{array}{l}\text { Automação Agropecuária com Foco em } \\
\text { Empreendimentos de Pequeno Porte }\end{array}$ & $\begin{array}{l}\text { Desenvolver metodologias, equipamentos e sistemas de automação } \\
\text { para investigar, caracterizar e melhorar a qualidade de produtos } \\
\text { gerados pela agricultura familiar de pequena escala, por meio da } \\
\text { instrumentação agropecuária. }\end{array}$ \\
\hline $\begin{array}{l}\text { Pesquisa, Desenvolvimento e Inovação } \\
\text { em Sistemas Inovadores de Produção } \\
\text { Agropecuária }\end{array}$ & $\begin{array}{l}\text { Disponibilizar conhecimentos, tecnologias, materiais e sistemas } \\
\text { tecnológicos para incrementar a base de conhecimentos estruturantes } \\
\text { e promover a inovação no agronegócio com foco em sistemas } \\
\text { inovadores de produção agropecuária. }\end{array}$ \\
\hline $\begin{array}{l}\text { Articulação Internacional para Avanço } \\
\text { da C, T \& I Voltada para o Agronegócio }\end{array}$ & $\begin{array}{l}\text { Desenvolver projetos de } \mathrm{P}, \mathrm{D} \& \mathrm{I} \text { em áreas estratégicas em conjunto com } \\
\text { instituições estrangeiras de ponta e transferir tecnologias para a } \\
\text { produção de alimentos, fibras e bioenergia para países em } \\
\text { desenvolvimento. }\end{array}$ \\
\hline $\begin{array}{l}\text { Recuperação das Organizações Estaduais } \\
\text { de Pesquisa Agropecuária - OEPAS Para } \\
\text { o Fortalecimento do Sistema Nacional } \\
\text { de Pesquisa Agropecuária }\end{array}$ & $\begin{array}{l}\text { Promover a reconstrução do Sistema Nacional de Pesquisa } \\
\text { Agropecuária, a partir do apoio ao fortalecimento das Organizações } \\
\text { Estaduais de Pesquisa Agropecuárias (OEPAs), tendo como base a } \\
\text { gestão estratégica e a articulação institucional em especial com a } \\
\text { Embrapa. }\end{array}$ \\
\hline
\end{tabular}

Fonte: MCT (2015).

Os programas do MCT abordam questões de inovação baseados na produção de novos conhecimentos. As tendências, na maioria dos casos, podem ser relacionadas a esses programas no que refere aos seus objetivos. A Figura 1, traz essa abordagem: 


\begin{tabular}{|l|}
\hline \multicolumn{1}{|c|}{ PROGRAMAS MCT PARA OS } \\
AGRONEGÓCIOS
\end{tabular}

Figura 1: Diagrama de relação entre os programas do MCT e as tendências tecnológicas do agronegócio Fonte: MCT (2015) e MAPA (2015).

Observando a possibilidade de criação do conhecimento, nesta relação estabelecida no Quadro 06, a partir da aplicabilidade dos programas, principalmente por terem relação com a maioria das tendências, denota-se o interesse do governo descrito por Negri e Cavalcante (2013), para com o desenvolvimento tecnológico, haja vista as possibilidades de impactos sobre os aspectos econômicos do país.

6.3 Maquinários agrícolas no Brasil: investimentos na produção e sua comercialização

Inseridas na Anfavea, constam 09 empresas, entre fabricantes de maquinários agrícolas e rodoviários, como informado no Quadro 06.

Quadro 6: Produtores de Maquinários Agrícolas e Rodoviários

\begin{tabular}{|c|c|c|c|c|}
\hline \multirow{2}{*}{ EMPRESAS } & \multicolumn{3}{|c|}{ MAQUINÁRIOS } \\
\cline { 2 - 5 } & $\begin{array}{c}\text { TRATORES DE } \\
\text { RODAS }\end{array}$ & $\begin{array}{c}\text { TRATORES } \\
\text { ESTEIRAS }\end{array}$ & COLHEITADEIRAS & RETROESCAVADEIRAS \\
\hline AGCO & $\mathrm{X}$ & & $\mathrm{X}$ & $\mathrm{X}$ \\
\hline Agrale & $\mathrm{X}$ & & $\mathrm{X}$ & $\mathrm{X}$ \\
\hline Caterpillar & & $\mathrm{X}$ & $\mathrm{X}$ & $\mathrm{X}$ \\
\hline $\begin{array}{c}\text { CNH } \\
\text { Industrial }\end{array}$ & $\mathrm{X}$ & $\mathrm{X}$ & $\mathrm{X}$ & \\
\hline John Deere & $\mathrm{X}$ & $\mathrm{X}$ & $\mathrm{X}$ & \\
\hline Komatsu & $\mathrm{X}$ & $\mathrm{X}$ & $\mathrm{X}$ \\
\hline Mahindra & & & & \\
\hline Valtra & & & & \\
\hline Volvo & & & & \\
\hline
\end{tabular}

Fonte: Anfavea (2015).

No Quadro 06 pode-se verificar que, do total de empresas, 6 delas estão vinculadas ao setor agrário e, destas, a metade delas atuam especificadamente no setor de máquinas agrícolas. Essa produção está concentrada nos estados de Rio Grande do Sul, São Paulo, Paraná e Goiás (ANFAVEA, 2015). 
A comercialização dos produtos agrícolas e rodoviário é feita a partir de concessionárias distribuídas por todo o país. O Quadro 07 indica o total de unidades por região:

Quadro 7: Produção de Maquinários Agrícolas e Rodoviários

\begin{tabular}{|c|c|c|c|c|c|}
\hline \multirow{2}{*}{ EMPRESAS } & \multicolumn{5}{|c|}{ REGIÕES BRASILEIRAS } \\
\cline { 2 - 6 } & NORTE & NORDESTE & SUDESTE & SUL & CENTRO-OESTE \\
\hline AGCO & 10 & 18 & 72 & 86 & 33 \\
\hline Agrale & 7 & 8 & 29 & 38 & 13 \\
\hline Caterpillar & 14 & 13 & 20 & 12 & 5 \\
\hline CNH Industrial & 19 & 24 & 84 & 109 & 76 \\
\hline John Deere & 16 & 23 & 71 & 88 & 5 \\
\hline Komatsu & 11 & 9 & 9 & 9 & 32 \\
\hline Valtra & 11 & 18 & 66 & 49 & 226 \\
\hline TOTAL & 88 & 113 & 351 & 391 & \\
\hline \multicolumn{7}{r|}{ TOTAL GERAL: 1.169} \\
\hline
\end{tabular}

O total de concessionárias no Brasil é de 1.169 unidades, sendo que 7,5\% estão inseridas na região Norte, 9,7\% na Nordeste, 30\% na Sudeste, 33,5\% na Sul e 19,3\% na Centro-Oeste. Em assim sendo, as regiões Sudeste e Sul possuem percentuais similares, porém a última pode ser considerada a região com maior quantidade de unidades de comercialização.

A comercialização desses produtos pode indicar o quantitativo de maquinários agrícolas que foram adquiridos nos últimos dois anos, o que significa maquinários com tecnologias mais avançadas em prol do aumento produtivo descrito por Oliveira et al. (2012). A quadro 8 faz esta quantificação, com especificações por região do Brasil e anos de 2013 e 2014.

Quadro 8: Produção de Maquinários Agrícolas e Rodoviários

\begin{tabular}{|c|c|c|c|c|c|c|c|c|c|c|}
\hline \multirow{2}{*}{\begin{tabular}{c} 
PROD. \\
\cline { 2 - 11 }
\end{tabular}} & \multicolumn{2}{|c|}{ NORTE } & \multicolumn{2}{|c|}{ NORDESTE } & \multicolumn{2}{c|}{ SUDESTE } & \multicolumn{2}{c|}{ SUL } & \multicolumn{2}{c|}{$\begin{array}{c}\text { CENTRO- } \\
\text { OESTE }\end{array}$} \\
\cline { 2 - 11 } & $\mathbf{1 3}$ & $\mathbf{1 4}$ & $\mathbf{1 3}$ & $\mathbf{1 4}$ & $\mathbf{1 3}$ & $\mathbf{1 4}$ & $\mathbf{1 3}$ & $\mathbf{1 4}$ & $\mathbf{1 3}$ & $\mathbf{1 4}$ \\
\hline $\begin{array}{c}\text { Cultivares } \\
\text { com Motor }\end{array}$ & 50 & 64 & 41 & 36 & 989 & 927 & 463 & 462 & 75 & 64 \\
\hline $\begin{array}{c}\text { Tratores de } \\
\text { Roda }\end{array}$ & 3.405 & 2.851 & 5.136 & 4.784 & 21.903 & 17.062 & 24.352 & 23.452 & 10.293 & 7.463 \\
\hline $\begin{array}{c}\text { Colheitadei- } \\
\text { pra }\end{array}$ & 238 & 218 & 394 & 306 & 912 & 547 & 4.351 & 3.248 & 2.644 & 2.129 \\
\hline \begin{tabular}{c} 
TOTAL \\
\hline
\end{tabular} & 3.693 & 3.133 & 5.571 & 5.126 & 23.804 & 18.536 & 29.166 & 27.162 & 13.012 & 9.656 \\
\hline
\end{tabular}

Fonte: Anfavea (2015).

Ao comparar os anos de 2013 e 2014, denota-se que o total de unidades comercializadas foi reduzido em 11.633 unidades. O Quadro 9 aborda os percentuais de contribuição por região, para o valor total da comercialização, nos respectivos anos.

Quadro 9: Percentuais de Composição na Comercialização em Unidades de Maquinários Agrícolas

\begin{tabular}{|c|c|c|c|c|c|c|c|c|c|c|c|}
\hline \multicolumn{10}{|c|}{ \% POR REGIÕES BRASILEIRAS } & \multirow{2}{*}{\multicolumn{2}{|c|}{ TOTAL POR REGIÃO (\%) }} \\
\hline \multicolumn{2}{|c|}{ NORTE } & \multicolumn{2}{|c|}{ NORDESTE } & \multicolumn{2}{|c|}{ SUDESTE } & \multicolumn{2}{|c|}{ SUL } & \multicolumn{2}{|c|}{$\begin{array}{c}\text { CENTRO- } \\
\text { OESTE }\end{array}$} & & \\
\hline 13 & 14 & 13 & 14 & 13 & 14 & 13 & 14 & 13 & 14 & 13 & 14 \\
\hline 4,9 & 8,1 & 7,4 & 8,1 & 31,6 & 29,1 & 38,8 & 42,7 & 17,3 & 15,2 & 100 & 100 \\
\hline
\end{tabular}

Fonte: Anfavea (2015). 
Os percentuais de composição por região no número total de comercializações foram mantidos na região Norte. Esse número sofreu um aumento nas regiões Nordeste e Sul e redução nas regiões Sudeste e Centro-Oeste. Mesmo assim, porém, a redução ocorrida na venda na região Sudeste trouxe maior influência ao valor final da produção em 2014, quando comparada a 2013, do que as outras regiões.

Essa redução na comercialização pode estar vinculada às dificuldades econômicas do país, mas é necessário compreender que a agricultura no Brasil, como explicaram Vian e Andrade Júnior (2010), para que consiga ser praticada para obtenção de resultados positivos na qualidade e produtividade, precisa mais e maiores investimentos em tecnologia.

Indica-se ainda que essas unidades produtoras realizem investimentos em pesquisas que buscam tornar seus produtos cada vez mais competitivos no mercado, através da oferta de elementos que supram as necessidades dos usuários. Desse modo, cabe descrever, no Quadro 10, os valores investidos no período de 2002 a 2012, intervalo temporal escolhido devido à disponibilidade de informação.

Quadro 10: Investimentos na Produção de Autoveículos e Maquinários Agrícolas e Rodoviários

\begin{tabular}{|c|c|c|c|}
\hline \multirow{2}{*}{ ANO } & \multicolumn{3}{|c|}{ INVESTIMENTOS (MILHÕES US\$) } \\
\cline { 2 - 4 } & AUTOVEÍCULOS & $\begin{array}{c}\text { MAQUINÁRIOS AGRÍCOLAS E } \\
\text { RODOVIÁRIOS - M.A.R. }\end{array}$ & $\begin{array}{c}\text { DIFERENÇA ENTRE OS } \\
\text { INVESTIMENTOS }\end{array}$ \\
\hline $\mathbf{2 0 0 2}$ & 976 & 66 & 910 \\
\hline $\mathbf{2 0 0 3}$ & 673 & 75 & 598 \\
\hline $\mathbf{2 0 0 4}$ & 739 & 81 & 658 \\
\hline $\mathbf{2 0 0 5}$ & 1.050 & 130 & 920 \\
\hline $\mathbf{2 0 0 6}$ & 1.451 & 121 & 1.330 \\
\hline $\mathbf{2 0 0 7}$ & 1.965 & 171 & 1794 \\
\hline $\mathbf{2 0 0 8}$ & 2.913 & 284 & 2.629 \\
\hline $\mathbf{2 0 0 9}$ & 2.518 & 203 & 2.315 \\
\hline $\mathbf{2 0 1 0}$ & 3.654 & 218 & 3.436 \\
\hline $\mathbf{2 0 1 1}$ & 4.971 & 368 & 4.603 \\
\hline $\mathbf{2 0 1 2}$ & 4.692 & 655 & 4.037 \\
\hline TOTAL & 25.602 & 2.372 & 23.230 \\
\hline
\end{tabular}

Fonte: Anfavea (2015).

As informações apresentadas destacam que, no período de 10 anos, os investimentos feitos na produção de autoveículos são maiores em $90,74 \%$ do que os destinados a maquinários agrícolas e rodoviários. Acredita-se que essa diferença está relacionada ao faro de o número de fabricantes de autoveículos ser maior que a outra produção, bem como pelo fato de a primeira atender um público maior que a segunda.

Ocorre, porém, que os investimentos feitos em M.A.R. aumentaram ano após ano, com exceção aos anos de 2006 e 2009. Isso pode ser suficiente ou não, pois, conforme Bambini et al. (2013), existem muitas tecnologias nos agronegócios para os mesmos problemas, enquanto lacunas estão à espera de soluções que representem inovações tecnológicas.

6.4 A inovação tecnológica futura dos agronegócios no Brasil e as indicações geográficas registradas para o setor 
Uma das maneiras encontradas pelo governo para incentivar as pesquisas e inovações tecnológicas no país e, consequentemente, o surgimento de novos conhecimentos, veio por meio da criação do Fundo Setorial de Agronegócio - FSA. O intuito era o de contribuir para o aumento dos recursos investidos em mecanismos que auxiliassem em melhorias da qualidade, do aumento produtivo e da competitividade do setor nas esferas internas e externas ao país. O FSA foi criado pela Lei Federal no 10.332/2001 e regulamentado pelo Decreto MCT no 4.157/2002 (MCT, 2002).

O Quadro 11, indica as sete diretrizes estratégicas referente ao contexto:

Quadro 11: Diretrizes do Fundo Setorial de Agronegócio

\begin{tabular}{|c|}
\hline DIRETRIZES FSA \\
\hline $\begin{array}{l}\text { 1- Estimular as ações de pesquisa, desenvolvimento e inovação que associem as práticas agropecuárias às questões } \\
\text { de saúde, segurança alimentar, gestão do agronegócio e economia, dentre outras. }\end{array}$ \\
\hline $\begin{array}{l}\text { 2- Fomentar a criação de startups em tecnologias inovadoras e o empreendedorismo de negócios para promover o } \\
\text { desenvolvimento do setor. Devem ser viabilizadas ações de fomento a projetos exclusivos de inovação tecnológica } \\
\text { de produtos, processos e know-how, assim como para a criação de novas empresas para atuarem no setor. }\end{array}$ \\
\hline $\begin{array}{l}\text { 3- Apoiar a pesquisa, o desenvolvimento, a avaliação e a validação de insumos e tecnologias agropecuárias, bem } \\
\text { como o desenvolvimento de métodos inovadores de gestão do agronegócio. }\end{array}$ \\
\hline $\begin{array}{l}\text { 4- Promover ações de pesquisa em recursos genéticos, melhoramento vegetal e animal, bem como desenvolver a } \\
\text { adaptação de espécies exóticas e a domesticação de espécies nativas de grande potencial econômico. }\end{array}$ \\
\hline $\begin{array}{l}\text { 5- Incentivar o desenvolvimento de tecnologias de processamento, embalagem e conservação de produtos de } \\
\text { origem animal e vegetal, com ênfase em padrões de qualidade e agregação de valor. }\end{array}$ \\
\hline $\begin{array}{l}\text { 6- Promover a pesquisa, o desenvolvimento e as inovações tecnológicas em sistemas de produção agropecuária } \\
\text { sustentável, com ênfase na redução de emissões de gases de efeito estufa, no monitoramento e mitigação de } \\
\text { impactos ambientais, e na racionalização de uso de insumos e recursos renováveis e não renováveis. }\end{array}$ \\
\hline $\begin{array}{l}\text { 7- Fomentar o desenvolvimento da agricultura familiar, da produção orgânica e de base agroecológica, e fortalecer } \\
\text { pesquisas em extensão tecnológica e modelos de assistência e organização de arranjos produtivos locais. }\end{array}$ \\
\hline
\end{tabular}

Fonte: CGEE (2013).

Essa relação de indicações é a proposta para o desenvolvimento de tecnologias futuras para os agronegócios. Essas tecnologias estão relacionadas a necessidades presentes e que, através de pesquisas utilizando recursos públicos do FSA, poderão ser supridas ou amenizadas. Esse desenvolvimento tecnológico contribui para uma produção sustentável e conformada às necessidades elencadas pela FAO (2012).

No contexto dos agronegócios, as Indicações Geográficas são importantes caracterizações que auxiliam na agregação de valor aos produtos com origem geográfica conhecida devido à tradição na qualidade e nos métodos de produção. Implicitamente, esse processo envolve conhecimento e, por isso, deve-se considerá-lo nesta pesquisa, no que tange a seus registros no Instituto Nacional da Propriedade Industrial - INPI.

O Quadro 12 representa a temporalidade dos depósitos dessas Indicações Geográficas, conforme o produto da região e a classificação do INPI relacionado aos agronegócios.

Quadro 12: Distribuição Temporal de Depósitos de Produtos com Indicações Geográficas

\begin{tabular}{|c|c|c|c|c|}
\hline \multirow{2}{*}{ ANO } & \multicolumn{4}{|c|}{ PRODUTOS } \\
\cline { 2 - 5 } & VINHOS/OUTROS & CACHAÇA & AGROPECUÁRIOS & CAFÉ \\
\hline 1997 & 1 & & 1 & 1 \\
\hline 1998 & 1 & & 1 & 1 \\
\hline 1999 & 1 & & 1 & \\
\hline $\mathbf{2 0 0 0}$ & 2 & & 1 & \\
\hline $\mathbf{2 0 0 1}$ & 1 & & & \\
\hline
\end{tabular}




\begin{tabular}{|c|c|c|c|c|}
\hline 2002 & 1 & & & 2 \\
\hline \multicolumn{5}{|l|}{2003} \\
\hline 2004 & & & 1 & \\
\hline 2005 & & & 1 & \\
\hline 2006 & 1 & 1 & & \\
\hline 2007 & & & 1 & 2 \\
\hline 2008 & 3 & & 1 & \\
\hline 2009 & 4 & 1 & 1 & 1 \\
\hline 2010 & 3 & & 2 & 1 \\
\hline 2011 & 2 & & 3 & \\
\hline 2012 & 4 & 1 & & 1 \\
\hline TOTAL & 23 & 3 & 14 & 9 \\
\hline \multicolumn{5}{|c|}{ TOTAL GERAL: 49} \\
\hline
\end{tabular}

Fonte: INPI (2015).

A frequência apresentada no quadro informa que, no Brasil, a maioria das Indicações Geográficas dos Agronegócios depositadas está relacionada a vinhos (47\%) e, em sequência, aparecem os produtos agropecuários (29\%), aparece o café (18\%) e aparece a cachaça (6\%). Isso implica a exposição do conhecimento agregado a esses produtos, expostos do modo como Welter e Castro (2013) consideram.

Com relação às Indicações registradas, o Quadro 13 traz a organização dos produtos relacionados aos agronegócios e seus respectivos anos de registro, no período de 1999 a 2014.

Quadro 13: Distribuição Temporal dos Registros de Produtos Vinculados aos Agronegócios com Indicações Geográficas

\begin{tabular}{|c|c|c|c|c|c|c|c|c|c|c|c|c|c|c|c|c|c|}
\hline \multirow[b]{2}{*}{ PRODUTO } & \multicolumn{16}{|c|}{ ANOS } & \multirow[t]{2}{*}{ Total } \\
\hline & 9 & 0 & 1 & 2 & 3 & 4 & 5 & 6 & 7 & 8 & 9 & 10 & 11 & 12 & 13 & 14 & \\
\hline Arroz & & & & & & & & & & & & 1 & & & & & 1 \\
\hline Biscoitos & & & & & & & & & & & & & & & 1 & & 1 \\
\hline Cacau & & & & & & & & & & & & & & 1 & & & 1 \\
\hline $\begin{array}{c}\text { Cachaça e } \\
\text { Outros }\end{array}$ & & & & & & & & & 1 & & & & & 1 & & 1 & 3 \\
\hline Café & & & & & & & 1 & & & & & & 1 & 1 & 2 & & 5 \\
\hline Cajuína & & & & & & & & & & & & & & & & 1 & 1 \\
\hline Camarão & & & & & & & & & & & & & 1 & & & & 1 \\
\hline $\begin{array}{c}\text { Carne } \\
\text { Bovina e } \\
\text { Derivados }\end{array}$ & & & & & & & & 1 & & & & & & & & & 1 \\
\hline $\begin{array}{c}\text { Carne Suína } \\
\text { e Presunto }\end{array}$ & & & & & & & & & & & 1 & & & & & & 1 \\
\hline Couro & & & & & & & & & & & 1 & & & & & & 1 \\
\hline Doces Finos & & & & & & & & & & & & & 1 & & & & 1 \\
\hline Frutas & & & & & & & & & & & 1 & & & & 1 & & 2 \\
\hline $\begin{array}{c}\text { Peixes } \\
\text { ornamen- } \\
\text { tais }\end{array}$ & & & & & & & & & & & & & & & & 1 & 1 \\
\hline Própolis & & & & & & & & & & & & & & 1 & & & 1 \\
\hline Queijo & & & & & & & & & & & & & 1 & 1 & 1 & & 3 \\
\hline $\begin{array}{l}\text { Algodão } \\
\text { Colorido }\end{array}$ & & & & & & & & & & & & & & 1 & & & 1 \\
\hline $\begin{array}{c}\text { Vinhos e } \\
\text { Outros }\end{array}$ & 1 & 1 & & 1 & 1 & & & & & & & 1 & & 6 & 1 & & 12 \\
\hline TOTAL ANO & 1 & 1 & 0 & 1 & 1 & 0 & 1 & 1 & 1 & 0 & 3 & 2 & 4 & 12 & 6 & 3 & 37 \\
\hline
\end{tabular}


Fonte: INPI (2015).

O Quadro 13 demonstra o registro de 37 Indicações Geográficas relacionadas aos agronegócios, dos quais contempla 17 tipos de produtos registrados. Destaca-se o ano de 2012, por apresentar maior número de registros de indicações de "vinhos e outros" - item que mais vezes foi registrado.

Para Gadotti (2005), a aprendizagem pode ocorrer em diferentes espaços, não se restringindo às salas de aula. Uma das contribuições tecnológicas úteis à sociedade refere-se ao comportamento social, que poderá influenciar a produção de bens e serviços, como indicam Vaz et al. (2009). Para Florida (2011), a criatividade dos indivíduos pode proporcionar ganhos financeiros. Tendo em vista as observações feitas pelos autores, as indicações geográficas podem ser um ponto de interseção entre esses elementos.

\section{CONSIDERAÇÕES FINAIS}

A produção dos agronegócios possui responsabilidade mundial devido ao aumento populacional e, assim, o crescimento das necessidades de alimentação dos indivíduos. Mediante a construção do conhecimento e a implantação da tecnologia, muitos avanços no aumento da quantidade de alimentos produzidos, bem como na qualidade dos produtos, foram alcançados.

Exige-se, porém, que haja a continuidade desse progresso. Essa exigência se põe ostensiva diante dos problemas que do setor que ainda não foram sanados ou que não conseguiram alguma redução ou melhoria. Ora, a exigência de continuidade de progresso implica a criação de novos conhecimentos para serem convertidos em novas tecnologias. Para isso, os incentivos governamentais funcionam como estímulos à pesquisa e ao desenvolvimento tecnológico do país, a exemplo dos incentivos proporcionados pelo Fundo Setorial de Agronegócios e também os proporcionados pelo BNDES.

Isso não significa que as iniciativas privadas devam estar a par da inovação do setor, pois os recursos utilizados significam investimentos feitos para esse tipo de desenvolvimento e podem significar uma diferenciação concorrencial da organização para com outras empresas do setor.

No que se refere ao conhecimento, identifica-se que, além das práticas de pesquisa, quando há uma conversão desse conhecimento em tecnologia, e esta é utilizada na propriedade rural, então passa a existir um compartilhamento de informações com um determinado número de proprietários e funcionários que consigam ter acesso à inovação - o que contribui para a qualificação dos usuários.

Considerando as contribuições da Companhia Nacional de Abastecimento (2010) e Cajavilca et al. (2014), pode-se afirmar que o desenvolvimento tecnológico, quando relacionado a alimentação da população em geral, deve continuamente ser desenvolvido para garantir uma produção sustentável. Para isso, as inovações devem ser acessíveis aos produtores, de modo a contribuir com a sua formação profissional continuada e a contínua melhoria produtiva. 


\section{REFERÊNCIAS}

Anfavea. (2015). Anuário da Indústria Automobilística Brasileira.

Araújo, M.J. (2013). Fundamentos de agronegócios. 4. ed. São Paulo: Atlas.

Bambini, M., Mendes, C.I., Moura, M.F. \& Oliveira, S.R.M. (2013). Software para agropecuária: panorama do mercado brasileiro. Parcerias Estratégicas, 18(36), Brasília.

Bazzo, W. A. (2012). Cultura científica versus humanística: A CTS é o Elo?. Revista Iberoamericana de Educación, (58).

BNDES - Banco Nacional de Desenvolvimento Econômico e Social. (2015). Agropecuária: Produtos e Programas. Disponível em: <http://www.bndes.gov.br/Site BNDES/bndes/bndes_pt/Areas_de_Atuacao/Agropecuaria/>. Acesso em: jan. 2015.

BNDES - Banco Nacional de Desenvolvimento Econômico e Social. (2015). Desembolsos Anuais na Comercialização, no País, de Máquinas e Equipamentos Nacionais, Finame, Finame Leasing e Finame Agrícola. Disponível em: <www.bndes.gov.br>. Acesso em: jan. 2015.

Borges, C.O., Mozzaquatro, P.M., Soares, R.M., Barcellos, C.B., Hartmann, M.L.B. \& Neubauer, V. S. (2013). Conhecimento e tecnologia: Biblioteca Virtual Disciplinas EAD. Revista Interdisciplinar de Ensino, Pesquisa e Extensão, 1(1), 141-150.

Brasil. (1988). Constituição Federal. Disponível em: <http://www4.planalto.gov.br/legislacao>. Acesso em: fev. 2015.

Brasil. (1996). Lei de Propriedade Intelectual do Brasil no 9.279. Disponível em: <http://www.planalto.gov.br/ccivil_03/leis/L9279.htm>. Acesso em: fev. 2015.

Bukowitz, W. R. \& Williams, R. L. (1999). The knowledge management fieldbook. Pearson Educations Limited.

Cajavilca, E.S.R., Marques, N.S, Melo, E.M., Santana, V.G., Sales, G.F. \& Lobo, R.S. (2014). Análise de patentes do mercado de alimentos industrializados no mundo com base na classificação "A" da WIPO. Cadernos de Prospecção, 7(4), 612-621.

Carvalho, M.G. (1997). Tecnologia, desenvolvimento social e educação tecnológica. Revista Educação \& Tecnologia, (1), 70-87.

Castells, M. (2000). A sociedade em rede. Tradução Roneide Venancio Majer e Klaus Brandini Gerhardt. São Paulo: Paz e Terra, 1-575.

CGEE - Centro de Gestão e Estudos Estratégicos. (2013). Diretrizes Estratégicas para o Fundo Setorial de Agronegócio - CT Agronegócio. Finep: Brasília, 1-35.

Companhia Nacional de Abastecimento. (2010). Custos de Produção Agrícola: a metodologia da Conab. Conab: Brasília, 1-60.

Coutinho, C. \& Lisbôa, E. (2011). Sociedade da informação, do conhecimento e da aprendizagem: desafios para a educação no século XXI. Revista de Educação, 18(1).

Cruvinel, P. E. (2008). Cenários do ambiente de atuação das instituições públicas e privadas de PD\&I para o agronegócio e o desenvolvimento rural sustentável - horizonte 2023. Relatório da Rede de Inovação e Prospecção Tecnológica para o Agronegócio - RIPA. Brasília, 1-100. 
DIAS, P. (2012). Comunidades de educação e inovação na sociedade digital. Educação, Formação \& Tecnologia, 5(2), 4-10.

Escola, J.J.J. (2005). Ensina a Aprender na Sociedade do Conhecimento. $4^{\circ}$ SOPCOM, 343-358.

Faléco, L.L. \& Jorge, C.F.B. (2017). O uso da informação e a sua aplicação como insumo estratégico para o agronegócio. Revista Inteligência Competitiva, 7(3), 95-117.

FAO - Food and Agriculture Organization of the United Nations. (2015). FAOSTAT. Disponível em: <http://faostat.fao.org/site/339/default.aspx>. Acesso em: jan. 2015.

Felipe, A.A.C. (2012). Reflexões sobre as mudanças sociais motivadas pelo desenvolvimento tecnológico: a necessidade de instituir uma reflexão ética na utilização das tecnologias da informação e comunicação (TIC). Biblionline, 8(2), 16-26.

Florida, R. (2011). A ascensão da classe criativa. L\&PM Editores, 1-452.

Gadotti, M. (2005). Informação, conhecimento e sociedade em rede: Que Potencialidades?. Educação, Sociedade \& Culturas, (23), 43-57.

INPI - Instituto Nacional da Propriedade Intelectual. (2015). Indicações geográficas reconhecidas. Disponível em: <www.inpi.org.br>. Acesso em: jan. 2015.

INPI - Instituto Nacional da Propriedade Intelectual. (2015). Depósitos de indicações geográficas por tipo de produto. Disponível em: <www. inpi.org.br>. Acesso em: jan. 2015.

Jongensen, M.H. (2012). Agricultural field machinery for the future - from an engineering perspective. Agronomy Research Biosystem Engineering, 109-113.

MAPA - Ministério da Agricultura, Pecuária e Abastecimento. (2015). Tendências tecnológicas para os agronegócios. Disponível em: <www.mapa.org.br>. Acesso em: jan. 2015.

MCT - Ministério da Ciência e Tecnologia. Diretrizes estratégicas do Fundo Setorial de Agronegócio. (2002). Disponível em: <http://www.mct.gov.br/index.php/ content/view/73420.html>. Acesso em: jan. 2015.

Nascimento, N.T.A., Medrado, S.S., Togni, F., Casemiro, I.P. \& São Pedro Filho, F. (2014). Gestão de tecnologia no agrobusiness: um estudo de caso na produção de abacaxi em Porto Velho, Brasil. Gestão, Inovação e Tecnologia, 4(3), 1076-1091.

Negri, F. \& Cavalcante, L.R. (2013). Sistemas de inovação e infraestrutura de pesquisa: considerações sobre o caso brasileiro. 24a Radar - tecnologia, produção e comércio exterior. IPEA- Diretoria de Estudos e Políticas Setoriais, de Inovação, Regulação e Infraestrutura. Brasília, 7-17.

Nowacki, R. \& Bachnik, K. (2016). Innovations within knowledge management. Journal of Business Research, 69, $1577-1581$.

Oliveira, M.A.C., Fonseca, D.F.M., Reis, C.V.S., Moreira, T.B. \& Pinheiro, A.A. (2012). Impulsoresposta do choque de inovação tecnológica à luz da teoria dos ciclos reais de negócios (RBC): o caso do setor agropecuário brasileiro. Revista de Economia e Agronegócio, 10(1), 1-28.

Redivo, A.R., Três, C. \& Ferreira, G.A. (2008). A tecnologia de informação aplicada ao agronegócio: um estudo sobre o "Sistema Agrogestor" nas fazendas do município de Sinop/MT. Revista Contabilidade \& Amazônia, 1(1), 43-52. 
Rocha, L.A., Khan, A.S., Lima, P.V. P.S. \& Sousa, E.P. (2014). A importância da política de inovação no desenvolvimento da agricultura brasileira. Estudos Sociedade e Agricultura, 22(1), 224-246.

Roco, M.C. \& Bainbridge, W.S. (2013). The new world of discovery, invention, and innovation: convergence of knowledge, technology and society. Journal of Nanoparticle Research, 15(9).

Santos, H.F. (2014). Modernização da agricultura e relação campo-cidade: uma Análise a partir do agronegócio cafeeiro no município de Alfenas - MG. Caminhos de Geografia, 15(51), 154-171.

Scudeler, M.A. \& Oliveira, M.C.S.C. (2013). A contribuição do sistema de patentes para o desenvolvimento econômico e tecnológico: uma análise sumária do perfil inovativo do país a partir dos depósitos de patentes perante o INPI. Anais. XXII Encontro Nacional do CONPEDI/ UNINOVE, 30-55.

Silva, H.C.H., Binotto, E. \& Vilpoux, O.F. (2016). Cooperação e compartilhamento de informação entre os atores sociais em um assentamento rural. Perspectiva em Gestão \& Conhecimento, 6(1), 89-108.

Silva, L.C.S., Kovaleski, J.L., Gaia, S. \& Francisco, A.C. Informação tecnológica: identificando tecnologias, vantagens e aplicações através do Banco Nacional e Internacional de Patentes. Holos, 1, 139-150.

Tavarayama, R. \& Martins, J. R. (2012). A sociedade da informação: possibilidades e desafios. Nucleos, 9(1), 267-276.

Vaz, C. R., Fagundes A.B. \& Pinheiro, N.A.M. (2009). O surgimento da ciência, tecnologia e sociedade (CTS) na educação: uma revisão. Anais. I Simpósio Nacional de Ensino de Ciência e Tecnologia, 98-116.

Vian, C.E.F. \& Andrade Júnior, A.M. (2010). Evolução histórica da indústria de máquinas agrícolas no mundo: origens e tendências. Anais. 48ㅇ SOBER, Campo Grande.

Werter, I.P. \& Castro, M.F. (2013). O direito à educação na sociedade da informação: a necessidade da inclusão digital para a inclusão social. Unoesc International Legal Seminar, 2(1), 143-155.

Zhou, Q., Lou, J., Xie, F. \& Liu, Q. (2011). The method research on optimum selection of agricultural machinery. International Journal of Digital Content Technology and its Applications, 5(6), 334337. 